\title{
Aplicabilidade da distribuição GEV ao estudo da precipitação máxima diária anual na região sul de Minas Gerais
}

Alisson Souza de Oliveira ${ }^{1}$

Carlos Rogério de Mello²

Camila Silva Franco ${ }^{3}$

Rosângela Francisca de Paula Vitor Marques ${ }^{4}$

Antônio Marciano da Silva ${ }^{5}$

\section{Resumo}

A precipitação é um dos elementos climáticos de grande influência nas atividades humanas, sendo o estudo de eventos extremos de grande importância no dimensionamento de obras hidráulicas. A distribuição de probabilidades Generalized Extreme Value (GEV) tem sido aplicada à hidrologia para estimativa de eventos de precipitação máxima diária anual, associados a frequências extremas (elevados tempos de retorno). Neste estudo, objetivou-se analisar a distribuição GEV, ajustada pelos métodos dos Momentos (MM), Máxima Verossimilhança (MMV) e Momentos-L (ML), a séries históricas de precipitação máxima diária anual de oito estações pluviométricas na região Sul do estado de Minas Gerais. Os resultados mostraram que o método ML proporcionou melhor ajuste da distribuição GEV, sendo o único que proporcionou adequação estatística das oito séries históricas, segundo teste de Filliben. As precipitações máximas diárias anuais para as estações de Poços de Caldas e Silvanópolis, que apresentaram as situações mais críticas, variaram entre 167 mm e 297 mm, entre os tempos de retorno de cinquenta e quinhentos anos, demonstrando a potencialidade de tragédias relacionadas a eventos extremos de precipitação nestas áreas. Já as menores precipitações ocorreram nas estações de Camanducaia e Monte Sião, variando entre 103 mm e 174 mm, para o mesmo intervalo de tempos de retorno. De maneira geral, a distribuição GEV ajustada pelos métodos MV e, principalmente, ML, apresentam-se como alternativa para estimativa da precipitação máxima diária anual para diferentes tempos de retorno.

Palavras-chave: Distribuição de probabilidade. Métodos de ajuste. Eventos de chuva extremos. Sul de Minas Gerais.

\section{Introdução}

O conhecimento dos aspectos estatísticos das precipitações máximas apresenta interesse de ordem técnica por sua frequente aplicação aos projetos de obras hidráulicas tais como vertedores de barragens, dimensionamento de canais, terraços e definição de obras de desvio de cursos d' água.

\footnotetext{
${ }^{1}$ Universidade Federal de Lavras, Doutorando em Recursos Hídricos em Sistemas Agrícolas. Lavras, Minas Gerais, Brasil. alissonso@ hotmail.com. (035) 3829-1684. UFLA - Cx. P. 3037, Lavras, Minas Gerias, CEP 37200-000.

${ }^{2}$ Universidade Federal de Lavras, Prof. Dr. Departamento de Engenharia Agrícola. Lavras, Minas Gerais, Brasil. crmello@deg.ufla.br. (035) 3829-1452. UFLA - Cx. P. 3037, Lavras, Minas Gerias, CEP 37200-000.

${ }^{3}$ Universidade Federal de Lavras, Doutoranda em Recursos Hídricos em Sistemas Agrícolas. Lavras, Minas Gerais, Brasil. camilasilvafranco@yahoo.com.br. (035) 3829-1684. UFLA - Cx. P. 3037, Lavras, Minas Gerias, CEP 37200-000.

${ }^{4}$ Universidade Federal de Lavras, Doutoranda em Recursos Hídricos em Sistemas Agrícolas. Lavras, Minas Gerais, Brasil. roeflorestal@ hotmail.com. (035) 3829-1684. UFLA - Cx. P. 3037, Lavras, Minas Gerias, CEP 37200-000.

5Universidade Federal de Lavras, Prof. Dr. Titular. Lavras, Minas Gerais, Brasil. marciano@deg.ufla.br (035) 3829-1386. UFLA - Cx. P. 3037, Lavras, Minas Gerias, CEP 37200-000.
} 
A distribuição de probabilidades generalizada de valores extremos (GEV) tem facilitado muitas aplicações da engenharia hidrológica devido à sua elevada precisão, sendo utilizada na modelagem de eventos extremos naturais como "cheias" (QUEIROZ, 2006), "chuvas" (BLAIN, 2011), "velocidade do vento" (BAUTISTA, 2002) e "outros extremos" (MARTINS; STEDINGER, 2000). Segundo Raynal (1997), a GEV é sempre uma interessante alternativa para a investigação da estatística de valores extremos, o que é corroborado por Nadarajah e Choi, (2007) quando afirmam que a GEV possui toda a flexibilidade contida em seus casos particulares. El Adlouni et al.,(2007), Pujol et al.,(2007), Méndez (2007), Furió e Meneu (2010) e Cannon (2010) utilizaram essa distribuição de probabilidades em estudos associados à frequência com que ocorrem os elementos meteorológicos extremos, os quais normalmente apresentam-se assintoticamente distribuídos, constatando boa precisão nos ajustes conduzidos.

O Método dos Momentos pode ser utilizado para estimativa dos parâmetros da distribuição GEV, apresentando como vantagem sua simplicidade. Porém, devido a constante presença de amostras com pequeno número de dados, seus estimadores normalmente apresentam atributos inferiores aos de outros métodos. O método da Máxima Verossimilhança é considerado aquele com maior eficiência por proporcionar os estimadores com maior plausibilidade possível, porém, em alguns casos, sua maior eficiência é apenas assintótica, o que faz com que sua aplicação a amostras de pequeno tamanho produza estimadores de qualidade comparável ou inferior a outros métodos. Além destes métodos, há ainda o método dos momentos ponderados por probabilidades, conhecido como Momentos-L, o qual pode produzir estimadores de parâmetros comparáveis, em qualidade, àqueles gerados pela Máxima Verossimilhança, com a vantagem de exigirem um menor esforço computacional (NAGHETTINI; PINTO, 2007).

O sul de Minas Gerais é uma das principais regiões econômicas do estado, possuindo uma agropecuária notadamente forte, bem como atividades industriais crescentes. Contudo, em praticamente todos os períodos de chuva, que ocorrem durante o verão, à localidade sofre com problemas associados a inundações provocados pela ocorrência de chuvas extremas, vindo a causar, além de alagamentos, deslizamentos de terra e interrupções em estradas rurais. Assim, estudos que venham a contribuir com uma estimativa mais exata possível das precipitações máximas, são fundamentais para nortear projetos que visem conter erosão, como dimensionamentos hidráulicos de canais escoadouros, barragens, drenagens urbanas e de estradas. Estudos que, por fim, gerem subsídios técnicos importantes para a engenharia hidráulica.

Diante do exposto, objetivou-se neste trabalho, verificar a aplicabilidade da distribuição GEV aplicada ao estudo da precipitação máxima diária anual, em localidades da região Sul de Minas Gerais, notadamente aquelas próximas ou sob influência da Serra da Mantiqueira.

\section{Material e Métodos}

\subsection{Base de dados e localização das estações pluviométri- cas}

O trabalho foi desenvolvido a partir de dados de precipitação máxima diária anual, obtidos junto à Agência Nacional de Águas (ANA/HIDROWEB), de oito estações pluviométricas localizadas na região sul do estado de Minas Gerais, pertencentes às Unidades de Planejamento e Gestão dos Recursos Hídricos (UPGRH), GD3, GD5, GD6 e Rio Piracicaba/Jaguari. 0 código, a localização, a altitude e o período analisado de cada estação estão apresentados na Tabela 1. 
Tabela 1. Estações pluviométricas, localização geográfica, altitude e série histórica utilizadas neste estudo.

\begin{tabular}{cccccc}
\hline Estações & Código & Latitude & Longitude & Altitude (m) & Período \\
\hline Andradas & 2246051 & $-22^{\circ} 04^{\prime} 00$ & $-46^{\circ} 34^{\prime} 00$ & 900 & $1941-1965$ \\
Camanducaia & 2246057 & $-22^{\circ} 45^{\prime} 36$ & $-46^{\circ} 08^{\prime} 49$ & 1040 & $1975-2004$ \\
Careaçu & 2245074 & $-22^{\circ} 03^{\prime} 10$ & $-45^{\circ} 41^{\prime} 56$ & 812 & $1942-2007$ \\
Machado & 2145033 & $-21^{\circ} 40^{\prime} 00$ & $-45^{\circ} 55^{\prime} 00$ & 873 & $1968-2008$ \\
Maria da Fé & 2245088 & $-22^{\circ} 18^{\prime} 53$ & $-45^{\circ} 22^{\prime} 23$ & 1200 & $1975-2008$ \\
Monte Sião & 2246055 & $-22^{\circ} 26^{\prime} 00$ & $-46^{\circ} 34^{\prime} 00$ & 980 & $1942-1962$ \\
Poços de Caldas & 2146048 & $-21^{\circ} 47^{\prime} 00$ & $-46^{\circ} 55^{\prime} 00$ & 1150 & $1961-1980$ \\
Silvanópolis & 2245089 & $-22^{\circ} 02^{\prime} 03$ & $-45^{\circ} 50^{\prime} 07$ & 900 & $1975-2008$ \\
\hline
\end{tabular}

Fonte: Elaboração dos autores.

Na Figura 1, pode-se visualizar a distribuição espacial de cada estação dentro do estado de Minas Gerais e na região Sul de Minas.

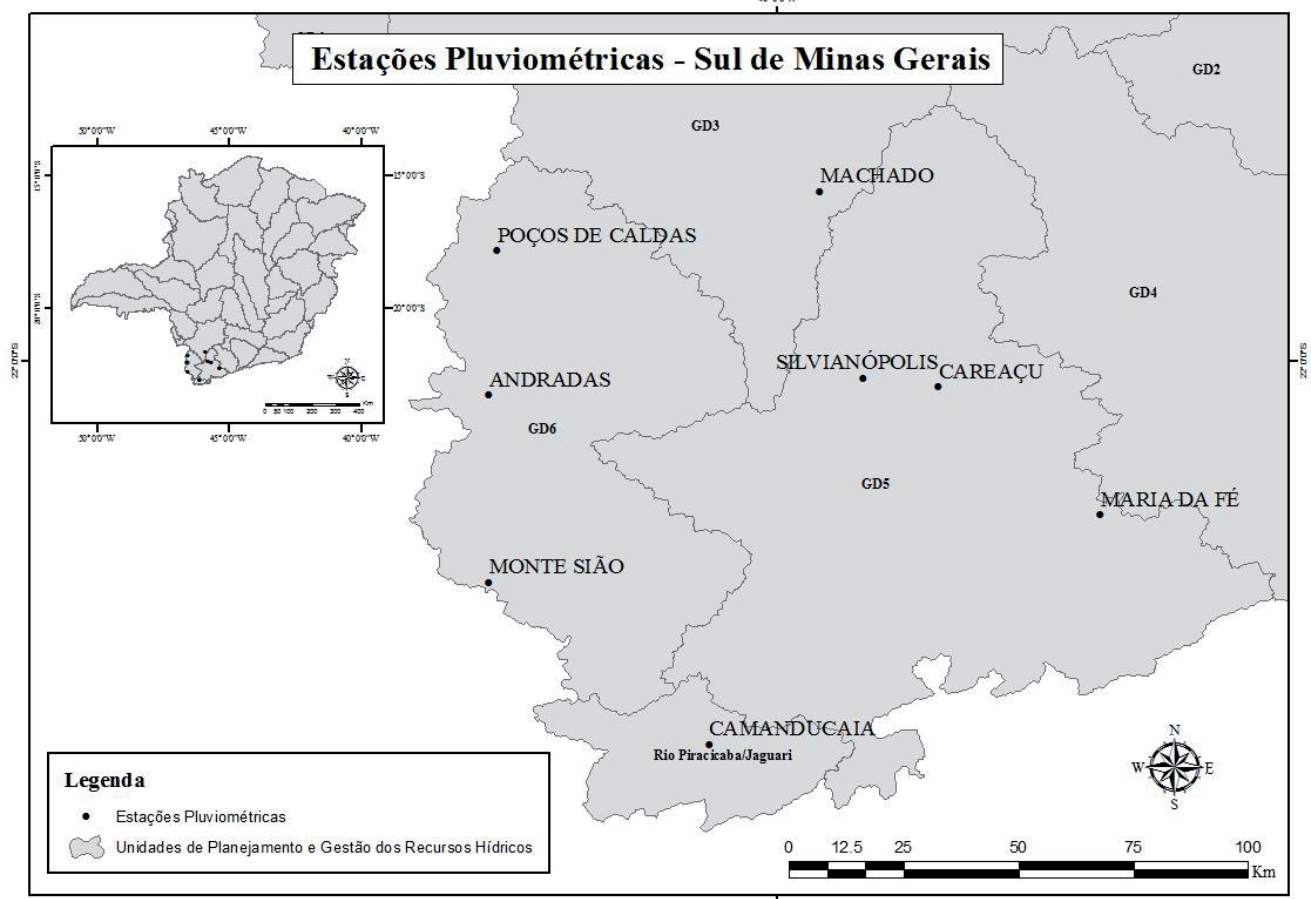

Figura 1. Distribuição espacial e localização das estações pluviométricas aplicadas ao estudo na região sul do estado de Minas Gerais.

Fonte: Elaboração dos autores.

\subsection{Distribuição de probabilidades GEV e metodologias de estimativa de seus parâmetros}

A função de distribuição generalizada de valores extremos (GEV), que engloba as três formas assintóticas de distribuição de valores extremos em uma única forma, Gumbel (Tipo I), Fréchet (Tipo II) e Weibull (Tipo III), vem sendo utilizada para representar a distribuição de valores extremos em diferentes campos, principalmente em estudos de precipitação máxima diária anual (Hartmann, 2011). Sua Função Densidade Probabilidade é dada por: 


$$
\mathrm{FDP}=\mathrm{f}(\mathrm{x})=\frac{1}{\sigma} \cdot\left[1-\xi \cdot\left(\frac{\mathrm{x}-\mu}{\sigma}\right)\right]^{\left(\frac{1+\xi}{\xi}\right)} \cdot \exp \left\{-\left[1-\xi \cdot\left(\frac{\mathrm{x}-\mu}{\sigma}\right)\right]^{\frac{1}{\xi}}\right\}
$$

O modelo apresenta três parâmetros, sendo que, $\sigma$ e são, os parâmetros de forma, escala e posição, respectivamente. Onde o parâmetro é quem determina a forma da distribuição, quando:

$\xi<0$, a GEV representa a forma assintótica de valores mínimos (Tipo III) e existe apenas para $\mathrm{x}<\frac{(\sigma-\mu)}{\xi}$. Se $\xi>0$, a GEV representa uma distribuição Tipo II (Fréchet), definida para $\mathrm{x}>\frac{(\sigma-\mu)}{\xi}$. Se $\xi=0$, tem-se a Distribuição Gumbel (Tipo I).

De posse dos valores numéricos dos parâmetros e considerando-se a frequência de excedência, a estimativa da precipitação máxima associada a um tempo de retorno é dada por:

$$
\mathrm{x}_{\mathrm{TR}}=\mu+\left(\begin{array}{l}
\sigma \\
\zeta
\end{array}\right)\left\{1-\left[-\ln \left(1-\frac{1}{\mathrm{TR}}\right)\right]^{\zeta}\right\}
$$

Em que $X_{T R}$ é o valor da variável hidrológica associada a um tempo de retorno em anos.

O método dos Momentos (MM) consiste em igualar os momentos amostrais aos populacionais. 0 resultado dessa operação produzirá as estimativas dos parâmetros da distribuição de probabilidades em questão. A distribuição GEV apresenta três momentos estatísticos, respectivamente, de $1^{a}, 2^{a}$ e $3^{a}$ ordens:

$$
\begin{gathered}
\mathrm{E}[\mathrm{x}]=\mathrm{x}=\mu+{ }_{\xi}^{\sigma} \cdot[1-\Gamma(1+\xi)] \\
\operatorname{Var}(\mathrm{x})=\left(\begin{array}{l}
\sigma \\
\xi
\end{array}\right)^{2} \cdot\left[\Gamma(1+2 \cdot \xi)-\Gamma^{2}(1+\xi)\right] \\
\gamma=C A=(\sin a l \text { de } \xi) \cdot\left[\frac{-\Gamma(1+3 \cdot \xi)+3 \cdot \Gamma(1+\xi) \cdot \Gamma(1+2 \cdot \xi)-2 \cdot \Gamma^{3}(1+\xi)}{\left[\Gamma(1+2 \cdot \xi)-\Gamma^{2}(1+\xi)\right]^{3}}\right]
\end{gathered}
$$

O método da Máxima Verossimilhança consiste de uma metodologia desenvolvida por Fisher em 1922 (Naghettini e Pinto, 2007), no qual se busca a maximização da probabilidade (plausibilidade) de um parâmetro representar uma população, maximizando a densidade conjunta dos elementos amostrais. A função de verossimilhança é matematicamente definida pelo produtório das densidades de cada valor amostral, sendo este dado por x1, x2, x3, etc, ou seja:

$$
L=f(x 1) \times f(x 2) \times f(x 3) \ldots f(x n)=\prod_{i=1}^{n} f\left(x_{i}\right)
$$

Assim, a máxima verossimilhança consiste em encontrar o ponto de máximo da função acima, derivando-a em relação a cada um dos seus parâmetros, e igualando-se a zero. Para facilitar a solução matemática do ponto de máximo, é necessário linearizar a equação 6 , obtendo-se a função logaritmo de verossimilhança $(\log (L))$ :

$$
\log (\mathrm{L})=\log (\mathrm{x} 1)+\log \mathrm{f}(\mathrm{x} 2)+\log (\mathrm{x} 3)+\cdots+\log f(\mathrm{xi})=\sum_{\mathrm{i}=1}^{\mathrm{A}} \log (\mathrm{x} 1)
$$


A distribuição GEV possui a FUNÇÃO DENSIDADE DE PROBABILIDADE conforme equação 1, logo, aplicando-se os conceitos de máxima verossimilhança, chega-se a seguinte equação:

$$
\mathrm{L}(\mu, \sigma, \xi)=\prod_{\mathrm{i}=1}^{\mathrm{n}} \mathrm{f}\left(\mathrm{x}_{\mathrm{i}}\right)=\frac{1}{\sigma^{\mathrm{n}}} \cdot \prod_{\mathrm{i}=1}^{\mathrm{n}}\left\{\left[1+\xi \cdot\left(\frac{\mathrm{x}_{\mathrm{i}}-\mu}{\sigma}\right)\right]^{-\left(\frac{1+\xi}{\xi}\right)}\right\} \exp \left\{\sum_{\mathrm{i}=1}^{\mathrm{n}}\left\{-\left[1+\xi \cdot\left(\frac{\mathrm{x}_{\mathrm{i}}-\mu}{\sigma}\right)\right]^{-\frac{1}{\xi}}\right\}\right\}
$$

O logaritmo da função verossimilhança produz:

$$
1(x ; \xi, \sigma, \mu)=\sum_{i=1}^{n}\left\{-\ln (\sigma)-\left(\frac{1+\xi}{\xi}\right) \cdot \ln \left[1+\xi \cdot\left(\frac{x_{i}-\mu}{\sigma}\right)\right]-\left[1+\xi \cdot\left(\frac{x_{i}-\mu}{\sigma}\right)\right]^{-\frac{1}{\xi}}\right\}
$$

Derivando a equação 9 em relação aos respectivos parâmetros e fazendo-se uma série de operações algébricas, chega-se ao seguinte sistema de equações:

$$
\begin{aligned}
& \sigma \cdot \sum_{\mathrm{i}=1}^{\mathrm{n}}\left(\begin{array}{c}
1+\xi-\mathrm{y}_{\mathrm{i}}^{-}{ }^{-} \xi \\
\sigma
\end{array}\right)=0 \\
& -\frac{n}{\sigma}+\frac{1}{\sigma^{2}} \cdot \sum_{i=1}^{n}\left\{\begin{array}{c}
\left(x_{i}-\mu\right) \cdot\left[(1+\xi)-y_{i}^{-1} \xi\right] \\
y_{i}
\end{array}\right\}=0
\end{aligned}
$$

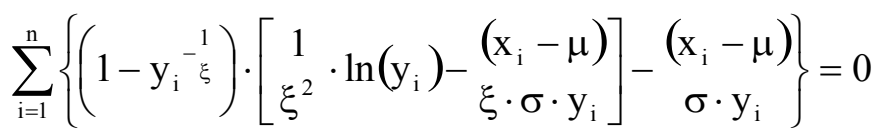

Neste caso, tem-se:

$$
\mathrm{y}_{\mathrm{i}}=\left[1+\xi \cdot\left(\frac{\mathrm{x}_{\mathrm{i}}-\mu}{\sigma}\right)\right]
$$

O método dos Momentos L consiste de uma abordagem que permite estimar os parâmetros de uma distribuição de probabilidades com base em momentos estatísticos de ordem superior a três, podendo-se, em casos de pequenas amostras, ajustar a distribuição com maior precisão do que o Método da Máxima Verossimilhança.

Desta forma, foram introduzidos os conceitos de momentos ponderados por probabilidades, apresentando a seguinte expressão para estimativa dos momentos amostrais:

$$
\hat{\alpha_{s}}=\frac{1}{N} \cdot \sum_{i=1}^{N} \frac{\left(\begin{array}{c}
N-i \\
s
\end{array}\right)}{N-1}\left(x_{i}\right.
$$

Em que $\mathrm{N}$ é o tamanho da amostra, s é um número inteiro que varia de 0 a 3 e Xi a variável hidrológica em questão. Os termos entre parênteses são obtidos pela análise combinatória. 
Segundo Hosking (1990), os momentos estimados pela equação 12 podem ser linearizados uma vez que, nesta forma, os momentos são de difícil aplicação para modelagem (caracterização da forma e escala) de uma distribuição de probabilidades. Assim, os momentos podem ser estimados pelas equações abaixo, sendo conhecidos como Momentos - L:

$$
\begin{aligned}
& \lambda_{1}=\alpha_{0} \\
& \lambda_{2}=\alpha_{0}-2 \cdot \alpha_{1} \\
& \lambda_{3}=\alpha_{0}-6 \cdot \alpha_{1}+6 \cdot \alpha_{2} \\
& \lambda_{4}=\alpha_{0}-12 \cdot \alpha_{1}+30 \cdot \alpha_{2}-20 \cdot \alpha_{3}
\end{aligned}
$$

O momento L é equivalente à média. O coeficiente de variação, o qual está associado ao parâmetro de escala, é dado por:

$$
\tau=\frac{\lambda_{2}}{\lambda_{1}}
$$

Os coeficientes de assimetria e curtose são obtidos respectivamente, por:

$$
\begin{gathered}
\tau_{3}=\begin{array}{l}
\lambda_{3} \\
\lambda_{2}
\end{array} \\
\tau_{4}=\begin{array}{l}
\lambda_{4} \\
\lambda_{2}
\end{array}
\end{gathered}
$$

A estimativa dos parâmetros da distribuição GEV aplicando-se os conceitos dos momentos L, chegam-se as seguintes equações:

$$
\hat{\xi}=(-1) \cdot\left(7,859 \cdot C+2,9554 \cdot C^{2}\right)
$$

Em que:

$$
\begin{aligned}
& \mathrm{C}=\begin{array}{c}
2 \\
3+\tau_{3}-\begin{array}{c}
\ln (2) \\
\ln (3)
\end{array}
\end{array} \\
& \tau_{3}=-3+\begin{array}{c}
2 \cdot\left(1-3^{-\xi}\right) \\
\left(1-2^{-\xi}\right)
\end{array} \\
& \hat{\sigma}=\begin{array}{c}
\lambda_{2} \cdot \xi \\
\Gamma(1+\xi) \cdot\left(1-2^{-\xi}\right)
\end{array} \\
& \hat{\mu}=\lambda_{1}-\frac{\sigma}{\xi} \cdot[1-\Gamma(1+\xi)]
\end{aligned}
$$

\subsection{Teste de Aderência de Filliben}

Filliben (1975) propõe um teste de normalidade baseado na correlação linear entre as estatísticas observadas ordenadas e os valores esperados de uma distribuição normal. Nesse teste, segundo Naghettini e Pinto (2007), dada uma amostra de tamanho $n(x 1, x 2, x 3, \ldots, x n)$, de uma variável aleatória $x$, e posta à hipótese nula de que a amostra foi extraída de uma população cuja distribuição 
de probabilidades é $F x(x)$, a estatística do teste de aderência de Filliben é construída com base no coeficiente de correlação linear $r$ entre as observações ordenadas de forma crescente $(x 1, x 2, x 3, \ldots$ , xn) e os quantis teóricos (w1, w2, w3, ... wn), os quais são calculados pela seguinte equação:

$$
\mathrm{wi}=\mathrm{F}_{\mathrm{x}}^{-1}(\mathrm{qi})
$$

Em que wi é o quantil teórico na ordem de classificação i, $F_{x}^{-1}$ : é a inversa da distribuição de probabilidades considerada e qi é a probabilidade empírica correspondente à ordem de classificação i.

Assim, a estatística de teste r é expressa por:

$$
r_{\text {calc }}=\frac{\sum_{i=1}^{N}\left(x_{i}-\bar{x}\right)\left(w_{i}-\bar{w}\right)}{\sqrt{\sum_{i=1}^{N}\left(x_{i}-\bar{x}\right) \cdot \sum_{i=1}^{N}\left(w_{i}-\bar{w}\right)}}
$$

sendo $r$ a estatística de teste de Filliben; Xi é o valor da variável aleatória na ordem de classificação i, Wi é o valor do quantil teórico na ordem de classificação i, é a média da amostra da variável aleatória x e o é a média dos quantis teóricos.

A probabilidade empírica qi é definida pela seguinte equação:

$$
q i=\frac{i-a}{N+1-2 \cdot a}
$$

sendo qi a probabilidade empírica correspondente à ordem de classificação i; i é a ordem de classificação; N é o tamanho da amostra e a é a variável que depende da distribuição de probabilidades.

Para hipótese nula do teste de Filliben declara que a amostra foi extraída de uma população cuja distribuição de probabilidades é $\mathrm{F}(\mathrm{x})$. Desta maneira, a região de rejeição da hipótese $\mathrm{H}_{0}$, a um nível de significância $\alpha$, é formada pelos valores de $r$ inferiores ao valor crítico do $r_{\text {crítico, }}$ dado pela distribuição de probabilidades da estatística do teste.

sendo qi a probabilidade empírica correspondente à ordem de classificação i; i é a ordem de classificação; N é o tamanho da amostra e a é a variável que depende da distribuição de probabilidades.

Para hipótese nula do teste de Filliben declara que a amostra foi extraída de uma população cuja distribuição de probabilidades é $\mathrm{F}(\mathrm{x})$. Desta maneira, a região de rejeição da hipótese $\mathrm{HO}$, a um nível de significância $\alpha$, é formada pelos valores de $r$ inferiores ao valor crítico do rcrítico, dado pela distribuição de probabilidades da estatística do teste.

\section{Resultados e Discussão}

\subsection{Séries Temporais das Estações Pluviométricas}

Vicente e Nunes (2004), estudando eventos extremos de precipitação máxima diária anual em Campinas, no estado de São Paulo, conceituaram precipitações acima de $100 \mathrm{~mm}$ como eventos extremos. Analisando-se as séries históricas obtidas observou-se que, em todas as estações, ocorreram, em pelo menos um ano, precipitação máxima diária anual acima dos $100 \mathrm{~mm}$, caracterizando regiões estudadas como propensas a chuvas intensas. 
A estação de Poços de Caldas foi a que apresentou a maior incidência de eventos de precipitação máxima diária anual acima de 100 mm (50\% dos anos analisados). Esta condição, aliada ao fato de que as condições topográficas da região de Poços de Caldas são de relevo ondulado a fortemente ondulado, faz com que essa região seja consideravelmente susceptível aos desastres naturais relacionados às chuvas intensas, e estudos de frequência destes fenômenos devem ser realizados no sentido de melhorar as condições de prevenção via projetos hidráulicos adequadamente dimensionados. A estação Silvanópolis apresentou 26,5\% dos anos analisados com precipitações acima de $100 \mathrm{~mm}$, sendo a segunda estação mais atingida por este tipo de evento. Para as estações de Careaçu e Maria da Fé os eventos de precipitação acima de 100 mm não ultrapassaram os 10,6\% dos anos estudados enquanto que nas estações de Andradas e Machado, as precipitações extremas ocorreram em 16,7 e $17,6 \%$ dos anos, respectivamente. As estações de Camanducaia e Monte Sião foram as que menos sofreram com este tipo de evento, apresentando, em suas séries históricas, apenas 3,3\% e 5,6\% dos anos com precipitações acima dos $100 \mathrm{~mm}$.

\subsection{Comparação entre os métodos estimadores dos parâme- tros da distribuição de probabilidade GEV}

Analisando-se a Tabela 2, é possível perceber que o único método de estimação dos parâmetros da distribuição GEV que foi adequado às oito séries estudadas foi o dos Momentos-L (ML), segundo o teste de aderência de Filliben. Comparando-se os métodos que apresentaram os melhores resultados, tendo-se os valores $r^{2}$ calculados ( $\left.r^{2} \mathrm{cal}\right)$, MV e ML, constata-se que embora o método ML tenha sido adequado em todas as séries estudadas, este foi superior apenas para as estações de Camanducaia, Monte Sião, Machado e Poços de Caldas. As demais estações (Andradas, Careaçu, Maria da Fé e Silvanópolis) apresentaram melhores resultados quando o método MV foi aplicado. Comparando-se os valores de ${ }^{2}{ }^{2}$ cal entre os métodos de MV e ML, verifica-se que, com exceção das estações de Machado e Poços de Caldas, as quais não foram adequadas, segundo o teste de Filliben, ao método MV, que a diferença entre eles é muito pequena, variando de 0,0002 a 0,0018. Desta maneira, tanto o método da MV e ML produziram bom desempenho da distribuição GEV, sendo confirmado pelos elevados valores de ${ }^{2}$ cal, os quais variaram de 0,9333 a 0,9940 e 0,9693 a 0,9938, respectivamente, para os métodos MV e ML.

Resultado semelhante foi obtido por Blain (2011), trabalhando com séries de precipitação máxima diária anual em Campinas, concluindo que os métodos MV e ML apresentaram resultados semelhantes, indicando que ambos proporcionaram bons ajustes a distribuição de probabilidade GEV.

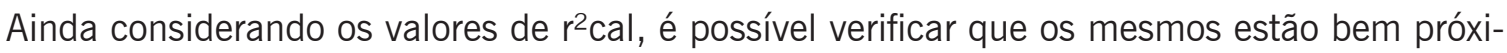
mos entre si quando se compara os métodos MM e MV, com exceção da estação de Careaçu, cuja diferença entre os métodos foi de 0,0018. Contudo, para esta estação, o método MV foi mais eficiente dentre os métodos testados, sendo superior inclusive ao ML. O melhor resultado do método MV em relação ao ML é explicado pelo fato do maior número de dados da série histórica da estação de Careaçu, totalizando sessenta e seis anos. Esta situação também foi verificada por Martins e

Stedinger (2001), que trabalhando com dados de vazão, verificaram que ocorre um ganho considerável na qualidade do ajuste da distribuição de probabilidade pela metodologia da máxima verossimilhança quando há incremento no tamanho da série histórica.

Verifica-se também que as estações de Machado e Poços de Caldas não foram adequadas, pelo teste de Filliben, aos métodos MM e MV. 
Tabela 2. Valores do teste de Filliben gerados pela distribuição de probabilidades GEV, ajustada pelos métodos dos Momentos (MM), Máxima Verossimilhança (MV) e Momentos-L (ML).

\begin{tabular}{ccccc}
\hline \multirow{2}{*}{ Estações } & MM & MV & ML & \multirow{2}{*}{$\mathbf{r}_{\text {tab }}$} \\
\cline { 2 - 4 } & $\mathbf{r}_{\text {cal }}{ }_{\text {cann }}$ & $\mathbf{r}_{\text {cal }}{ }^{2}$ & $\mathbf{r}_{\text {cal }}$ & \\
\hline Andradas & $0.9819^{\mathrm{A}}$ & $0.9836^{\mathrm{A}}$ & $0.9822^{\mathrm{A}}$ & 0.9670 \\
Camanducaia & $0.9846^{\mathrm{A}}$ & $0.9877^{\mathrm{A}}$ & $0.9879^{\mathrm{A}}$ & 0.9670 \\
Careaçu & $0.9872^{\mathrm{A}}$ & $0.9883^{\mathrm{A}}$ & $0.9865^{\mathrm{A}}$ & 0.9850 \\
Monte Sião & $0.9916^{\mathrm{A}}$ & $0.9923^{\mathrm{A}}$ & $0.9932^{\mathrm{A}}$ & 0.9670 \\
Machado & $0.9005^{\mathrm{NA}}$ & $0.9761^{\mathrm{NA}}$ & $0.9923^{\mathrm{A}}$ & 0.9790 \\
Maria da Fé & $0.9906^{\mathrm{A}}$ & $0.9923^{\mathrm{A}}$ & $0.9915^{\mathrm{A}}$ & 0.9594 \\
Poços de Caldas & $0.9304^{\mathrm{NA}}$ & $0.9333^{\mathrm{NA}}$ & $0.9693^{\mathrm{A}}$ & 0.9530 \\
Silvanópolis & $0.9900^{\mathrm{A}}$ & $0.9940^{\mathrm{A}}$ & $0.9938^{\mathrm{A}}$ & 0.9790 \\
\hline
\end{tabular}

A: valor de $r^{2}$ cal maior ou igual a $r^{2}$ tab - distribuição Adequada; NA: valor de $r^{2}$ cal menor que $r^{2}$ tab - distribuição Não Adequada.

Fonte: Elaboração dos autores.

A precisão do ajuste da distribuição de probabilidade GEV às frequências observadas da série de precipitação máxima diária anual em cada estação pode ser verificada na Figura 2. As estações de Andradas, Camanducaia, Careaçu e Monte Sião, apresentam o mesmo comportamento do ajuste da distribuição GEV, para os três métodos, MM, MV e ML. Contudo, para as estações de Machado e Poços de Caldas, apenas o método ML pôde ser considerado adequado e, portanto, com um bom ajuste aos dados de frequência observados. Para as estações de Maria da Fé e Silvanópolis, ocorreram uma pequena diferença, com uma maior aderência do método MV. 


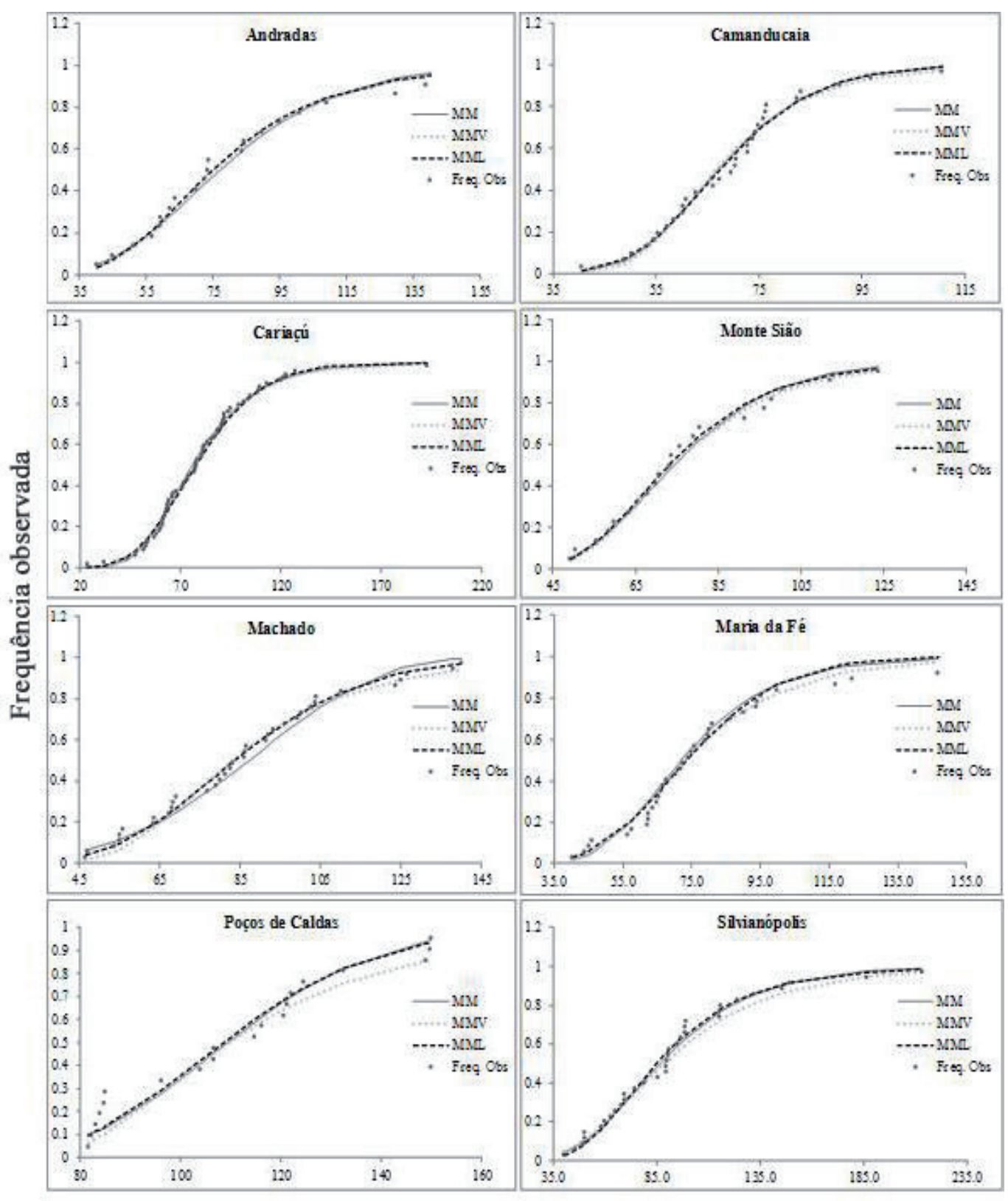

Precipitação máxima diária anual (mm)

Figura 2. Aderência da distribuição GEV às frequências observadas com base nos parâmetros estimados pelos Métodos dos Momentos (MM), Máxima Verossimilhança (MV) e Momentos L (ML).

Fonte: Elaboração dos autores.

Na Tabela 3 observam-se os parâmetros de ajuste da distribuição GEV obtidos pelos métodos estimadores, para cada série histórica. Verifica-se que os mesmos apresentam valores semelhantes entre si, o que significa dizer que todos eles proporcionaram à distribuição GEV um bom ajuste às séries de precipitação máxima diária anual, com exceção das estações de Machado e Poços de Caldas quando ajustadas pelos métodos dos MM e MV. 
Tabela 3. Parâmetros de ajuste da distribuição de probabilidade GEV gerados pelos Métodos dos Momentos (MM), Máxima Verossimilhança (MV) e Momentos L (ML).

\begin{tabular}{cccccccccc}
\hline \multirow{2}{*}{ Estações } & \multicolumn{3}{c}{ MM } & \multicolumn{1}{c}{$\mathbf{M V}$} & \multicolumn{4}{c}{$\mathbf{M L}$} \\
\cline { 2 - 10 } & $\boldsymbol{\sigma}$ & $\boldsymbol{\mu}$ & $\boldsymbol{\varepsilon}$ & $\boldsymbol{\sigma}$ & $\boldsymbol{\mu}$ & $\boldsymbol{\varepsilon}$ & $\boldsymbol{\sigma}$ & $\boldsymbol{\mu}$ & $\boldsymbol{\varepsilon}$ \\
\hline Andradas & 24.425 & 68.06 & -0.0606 & 23.328 & 67.78 & -0.0212 & 22.541 & 66.47 & -0.0546 \\
Camanducaia & 12.799 & 62.55 & -0.0892 & 12.092 & 62.34 & -0.0373 & 13.183 & 62.75 & 0.1255 \\
Careaçu & 21.340 & 68.51 & -0.0006 & 21.465 & 68.58 & -0.0087 & 21.750 & 68.99 & 0.0361 \\
Monte Sião & 17.054 & 68.12 & -0.0702 & 16.737 & 68.03 & -0.0531 & 16.201 & 67.18 & -0.0199 \\
Machado & 25.889 & 78.26 & -0.3635 & 21.153 & 75.32 & -0.0728 & 22.846 & 75.49 & 0.1268 \\
Maria da Fé & 17.694 & 65.56 & -0.0025 & 19.378 & 65.98 & -0.0806 & 17.235 & 65.35 & -0.0245 \\
Poços de & 21.961 & 101.45 & -0.1857 & 21.737 & 101.33 & -0.17234 & 21.686 & 100.79 & 0.1299 \\
Caldas & & & & & & & & & \\
Silvanópolis & 30.330 & 75.65 & -0.0005 & 33.322 & 76.39 & -0.08101 & 28.120 & 74.66 & -0.0751 \\
\hline
\end{tabular}

Fonte: Elaboração dos autores.

De maneira geral, o método ML foi o que proporcionou os melhores ajustes, além do fato de ter sido o único método em que todas as estações foram aprovadas pelo teste de Filliben, conforme já discutido anteriormente. Em geral, o método MV foi melhor para as séries em que o parâmetro de forma foi negativo, com exceção da estação de Careaçu, e ambos os métodos, apresentaram praticamente os mesmos valores dos parâmetros de escala e posição para positivo.

\subsection{Estimativa da precipitação máxima diária anual associa- da a diferentes tempos de retorno (TR)}

Para fins de identificação dos valores de precipitação máxima diária anual, estimados pela GEV cujo ajuste foi adequado, na Tabela 4 estão apresentados estes valores para os tempos de retorno de cinquenta, cem e quinhentos anos para as oito estações pluviométricas.

Considerando-se o método ML, percebe-se que as diferenças entre as precipitações máximas diárias anuais, estimadas pelos diferentes métodos, apresenta uma variação pequena entre si, para as estações de Andradas, Careaçu e Monte Sião. Comparando-se para estas estações os métodos da MV e ML, percebe-se que a máxima variação ocorreu para o tempo de retorno de quinhentos anos, sendo que na estação de Andradas o método MV estimou a maior precipitação assim como para as estações de Careaçu e Monte Sião.

As estações de Machado e Poços de caldas foram adequadas somente quando ajustadas pelo método dos ML e, portanto, apenas os valores associados a este ajuste foram apresentados. As demais estações, Camanducaia, Maria da Fé e Silvanópolis, apresentaram diferenças entre os métodos MV e ML um pouco mais acentuadas e em todos os casos o método MV estimou o maior valor da precipitação máxima diária anual. 
Tabela 4. Precipitação máxima diária anual estimada pela distribuição de probabilidade GEV, ajustada pelos métodos dos Momentos (MM), Máxima Verossimilhança (MV) e Momentos L (ML), para os tempos de retorno de 50, 100 e 500 anos.

\begin{tabular}{cccccccccc}
\hline \multirow{2}{*}{ Estações } & \multicolumn{3}{c}{ MM } & \multicolumn{3}{c}{ MV } & \multicolumn{3}{c}{ ML } \\
\cline { 2 - 10 } & $\mathbf{5 0}$ & $\mathbf{1 0 0}$ & $\mathbf{5 0 0}$ & $\mathbf{5 0}$ & $\mathbf{1 0 0}$ & $\mathbf{5 0 0}$ & $\mathbf{5 0}$ & $\mathbf{1 0 0}$ & $\mathbf{5 0 0}$ \\
\hline Andradas & 176 & 198 & $\mathbf{2 5 2}$ & 163 & 180 & 223 & 165 & 184 & 233 \\
Camanducaia & 122 & 135 & 169 & 113 & 123 & 147 & 103 & 109 & 120 \\
Careaçu & 152 & 167 & 201 & 154 & 169 & 206 & 148 & 161 & 190 \\
Monte Sião & 145 & 161 & 201 & 141 & 155 & 191 & 133 & 145 & 174 \\
Machado & - & - & - & - & - & 242 & 146 & 155 & 174 \\
Maria da Fé & 135 & 147 & 176 & 155 & 174 & 222 & 136 & 149 & 181 \\
Poços de Caldas & - & - & - & - & - & 343 & 167 & 176 & 193 \\
Silvanópolis & 194 & 215 & 264 & 229 & 262 & 346 & 202 & 229 & 297 \\
\hline
\end{tabular}

Fonte: Elaboração dos autores.

\section{Conclusões}

O método ML proporcionou melhor ajuste da distribuição GEV às séries históricas de precipitação máxima diária anual, sendo o único que apresentou adequação às oito séries históricas, segundo teste de Filliben.

Comparando-se os métodos, percebem-se comportamentos diferenciados para as estimativas da precipitação máxima diária anual. Quando os parâmetros foram estimados pela Máxima Verossimilhança, com exceção da estação de Andradas, houve uma estimativa superior da precipitação em todas as estações, para todos os tempos de retorno. Caso semelhante aconteceu quando os parâmetros foram ajustados pelo Método MM, em que ocorreu uma estimativa superior da precipitação em todas as estações, exceto na de Maria da Fé.

As precipitações máximas diárias anuais para as estações de Poços de Caldas e Silvanópolis, que apresentaram as situações mais críticas, variaram entre $167 \mathrm{~mm}$ e $297 \mathrm{~mm}$, entre os tempos de retorno de cinquenta e quinhentos anos. Já as menores precipitações ocorreram nas estações de Camanducaia e Monte Sião, variando entre 103 mm e 174 mm, para o mesmo intervalo de tempos de retorno.

De maneira geral, a distribuição GEV ajustada pelos métodos MV e, principalmente, ML, apresentam-se como opção para a estimativa da precipitação máxima diária anual para diferentes tempos de retorno. 


\section{Applicability GEV distribution for studies of maximum rain- fall in the region of south of Minas Gerais}

\section{Abstract}

The rainfall is one of the climates elements with great influence on human activities, and the studies of extremes events are very important in the sizing of hydraulic constructions. The probability distribution, GEV (Generalize Extreme Value), has been applied to hydrology for estimate the events of maximum daily annual rainfall, associated with extreme frequency (high return time). The objective of this paper is to analyze the GEV distribution, adjusted using the Moments Method (MM), Maximum Verisimilitude (MMV) and Moments-L (ML). For this, was used historical data from 8 rainfall stations located at south of Minas Gerais state. The results has showed that the ML method provided the best adjustment for the GEV distribution, being the only one that provided statistic adequacy for the 8 historical data by using Fiberfill test. The maximum daily annual rainfall for the Poços de Caldas and Silvianópolis station, where presented critical situation, ranged between 167 and $297 \mathrm{~mm}$, with the return time of 50 and 500 years, showing the potentiality for tragedies related with extreme events of rainfall in this areas. However, the lowest values occurred at Camanducaia and Monte Sião stations, ranging between 103 and $174 \mathrm{~mm}$ for the same return interval. Generally speaking, the GEV distribution adjusted using the MV, and mostly ML methods, are presented as an alternative for estimate maximum daily annual rainfall using different return times.

Keywords: Probability distribution. Adjustment methods. Extreme rainfall events. South of Minas Gerais.

\section{Referências bibliográficas}

BAUTISTA, E. A. L. A distribuição generalizada de valores extremos no estudo da velocidade máxima do vento em Piracicaba, SP. 2002. 47 p. Dissertação (Mestrado em Estatística e Experimentação Agronômica) - ESALQ/USP, Piracicaba, 2002.

FERREIRA, M.C. et al. Fatores qualitativos da ponta de energia hidráulica ADGA 110015 para pulverização agrícola. Engenharia Agrícola, Jaboticabal, v. 27, n. 2, p. 471-478, maio-ago. 2007.

BLAIN, G. C. Cento e vinte anos de totais extremos de precipitação pluvial máxima diária em Campinas, estado de São Paulo: análises estatísticas. Bragantia, Campinas, v. 70, n. 3, p. 722-728, 2011.

CANNON, A.J. A flexible nonlinear modeling framework for nonstationary generalized extreme value analysis in hydroclimatology. Hydrological Process, v. 24, p. 673-685, 2010.

EL ADLOUNI, S. et al. Generalized maximum likelihood estimators for the nonstationary generalized extreme value model. Water Resources Research, v. 43, p.1-13, 2007.

FILLIBEN J. J. The probability plot correlation coefficient test for normality. Technometrics, 17(1), pp. 111-117, 1975.

FURIÓ, D.; MENEU, V. Analysis of extreme temperatures for four sites across Peninsular Spain. Theoretical Applied Climatology, v. 24, p. 1-17, 2010.

HARTMANN, M.; MOALA, F. A.; MENDONÇA, M. A. Estudo das precipitações máximas anuais em presidente prudente. Revista Brasileira de Meteorologia, São José dos Campos, v. 26, n. 4, p. 561568, 2011. 
HOSKING, J.R.M. L - moments: analysis and estimation of Distributions using Linear Combinations of Order Statistics. Journal of Royal Statistical Society, v. 52, n. 1, p. 105-124, 1990.

MARTINS, E. S.; STEDINGER, J. R. Generalized maximum-likelihood generalized extreme-value quantile estimators for hydrologic data. Water Resources Research, Washington, v. 36, n. 3, p. 737$744,2000$.

MARTINS, E. S.; STEDINGER, J. R. Historical information in a generalized maximum likelihood framework with partial duration and annual maximum series. Water Resources Research, Washington, v. 37, n. 10, p. 2559-2567, 2001.

MÉNDEZ, F.J. et al. Analyzing Monthly Extreme Sea Levels with a Time-Dependent GEV Model. Journal of Atmospheric and Oceanic Technology. v. 24, p. 894-911, 2007.

NADARAJAH, S.; CHOI, D., Maximum daily rainfall in South Korea. Journal of Earth System Science, v. 116, p. 311-320, 2007.

NAGHETTINI, M.; PINTO, E. J. A. Hidrologia Estatística, CPRM, Belo Horizonte, 552 p. 2007.

PUJOL, N.; NEPPEL, L.; SABATIER, R. Regional tests for trend detection in maximum precipitation series in the French Mediterranean region. Hydrological Sciences Journal, v. 52, p. 952-973, 2007.

QUEIROZ, M. M. F.; CHAUDHAY, F. H. Análise de eventos hidrológicos extremos, usando-se a distribuição GEV e momentos L.H. Agrimbi, v. 10, n. 2, p. 381-389, 2006.

RAYNAL, J.A. Sobre el uso del dominio de atracción para la identificación de valores extremos para máximos. Ingenieria Hidrólica en Mexico, v. 12, p. 57-62, 1997.

VICENTE, A.K.; NUNES, L.H. Extreme precipitation events in Campinas, Brazil. Terrae, v. 1, p. 6062, 2004.

\section{Histórico editorial}

Recebido: 02/04/2013

Avaliação e copidesque: 08/04/2013 a 11/02/2014 